\title{
A Clinical Analysis of Microvascular Decompression Surgery with Sacrification of the Superior Petrosal Venous Complex for Trigeminal Neuralgia: A Single-Surgeon Experience
}

\author{
Efdal AKKAYA ${ }^{1}$, Zeki GOKCILㄹ ${ }^{2}$ Cem ERBAS ${ }^{3}$, Serhat PUSAT ${ }^{4}$, Zuleyha KAZAK BENGISUN ${ }^{5}$, Ersin ERDOGAN ${ }^{3}$ \\ ${ }^{1}$ Ufuk University, School of Medicine, Department of Neurology, Ankara, Turkey \\ ${ }^{2}$ Eastern Mediterranean University, Faculty of Health Sciences, Physiotherapy and Rehabilitation, Department of Neurology, Famagusta, \\ North Cyprus via Mersin 10, Turkey \\ ${ }^{3}$ Brain and Spine Surgery Center, Ankara, Turkey \\ ${ }^{4}$ Sultan Abdulhamid Han Education and Research Hospital, Department of Neurosurgery, Istanbul, Turkey \\ ${ }^{5}$ Ufuk University, School of Medicine, Department of Anesthesiology, Ankara, Turkey
}

Corresponding author: Efdal AKKAYA efdalakkaya@hotmail.com

\section{ABSTRACT}

AIM: To report the surgical outcomes in patients with trigeminal neuralgia (TN) who underwent microvascular decompression (MVD) with superior petrosal vein sacrification.

MATERIAL and METHODS: Data from 63 patients, whose information was obtained from a group of 113 patients who underwent surgery from 2008 to 2018, were reviewed retrospectively by the first author who was not part of the surgical team, and the pain conditions were evaluated objectively.

RESULTS: Following surgery, pain relief occurred in $84 \%$ of patients during the early postoperative period and in $69.8 \%$ of patients during long-term follow-up. The major offending vessel was the superior cerebellar artery.

CONCLUSION: MVD surgery, in particular for patients with typical pain, is one of the most effective treatment strategies for TN. Superior petrosal vein sacrification is a safe method that helps neurosurgeons to visualise the surgical area and perform a better work-up. Neurosurgeons should not be afraid to carry out superior petrosal vein sacrification.

KEYWORDS: Microvascular decompression, Superior petrosal vein, Surgery, Trigeminal neuralgia

\section{INTRODUCTION}

$\mathrm{T}$ Trigeminal neuralgia (TN) was first described in 1934 by Dandy as a disease caused by vascular compression of the trigeminal nerve $(2,4)$, and has been called the 'suicide disease' due to the severity of pain that ruins daily life. There are two types of TN: typical TN, characterised by sharp, stabbing, electrical pain (type 1), and atypical TN (type 2), with which patients complain of less intense, constant, dull or burning pain. In comparison with ablative procedures such as radiofrequency thermocoagulation, percutaneous balloon compression, or stereotactic radiosurgery, microvascular decompression surgery (MVD) is superior in pain relief (7). In this review, we aimed to evaluate our MVD surgery outcomes in light of the literature.

\section{MATERIAL and METHODS}

In the present retrospective study, we evaluated data from 63 patients whose information was obtained from a group of 113 patients who underwent MVD surgery from 2008 to 2018. Patients were called by the principal investigating neurologist
Efdal AKKAYA (10): 0000-0002-6656-0989

Zeki GOKCIL (1) : 0000-0002-0544-524X

Cem ERBAS (1): 0000-0001-7719-9271
Serhat PUSAT

(1) : $0000-0003-2412-2320$

Zuleyha KAZAK BENGISUN (D): 0000-0002-0731-2969

Ersin ERDOGAN
D : $0000-0003-1308-949 \mathrm{X}$ 
who was not part of the surgical team, and the pain conditions were evaluated objectively. All patients were diagnosed with trigeminal neuralgia according to criteria of the $2^{\text {nd }}$ and $3^{\text {rd }}$ editions of the International Classification of Headache Disorders (ICHD-2 and -3). Detailed medical history, duration of illness, previously performed treatment techniques, and presence of hypertension were recorded. All patients were refractory to medical therapy. All MVD surgery procedures were performed by one neurosurgeon (EE). Patients were questioned regarding the change in the severity of pain prior to and following surgery, which was classified using the Barrow Neurological Institute (BNI) Pain Intensity Scale Score (Table I) (17).

All patients underwent magnetic resonance imaging (MRI) prior to surgery to determine neurovascular conflict (Figure 1A, B), with 1.5 or 3T contrast-enhanced T1W, FLAIR, T2W, and 3D FIESTA (or 3D CISS) sequences being evaluated. MRI predicted neurovascular conflict in $83 \%$ of cases. Vascular compression status was better determined during surgical exploration in all cases. Moreover, all patients underwent computed tomography (CT) following surgery to control surgical complications and examine the surgical area.

During surgery, the trigeminal rhizotomy technique was performed in 4 cases with no neurovascular conflict. PTFE

Table I: Barrow Neurological Institute (BNI) Pain Intensity Scale Score (4)

\begin{tabular}{ll}
\hline Score & Description \\
\hline I & No pain, no medication. \\
\hline III & Occasional pain, not requiring medication \\
\hline IV & $\begin{array}{l}\text { Some pain, adequately controlled with medication } \\
\text { medication }\end{array}$ \\
\hline V & Severe pain / no pain relief \\
\hline
\end{tabular}

polymer Teflon ${ }^{\mathrm{TM}}$ pledgets were used as Teflon ${ }^{\mathrm{TM}}$ felt. In all patients with arterial compression and one patient with venous compression, Teflon ${ }^{\mathrm{TM}}$ felt was implanted between the offending vessel and the trigeminal nerve (Figure 2). With the exception of one, the superior petrosal vein was sacrificed in all patients.

\section{Surgical Technique}

Following the induction of anesthesia and intubation, a threepoint head-fixation device was applied and the patient was placed in the lateral decubitus position with appropriate pressure points and an axillary silicone roll. The neck was flexed slightly, with the chin approximately two finger-breadths from the sternum. The head was rotated 10 degrees away from the affected side. The vertex was kept parallel to the floor to keep the $7^{\text {th }}$ and $8^{\text {th }}$ cranial nerve complex at a more inferior position in relation to the trigeminal nerve. The retrosigmoid approach was performed as described previously by our own experience (6). An incision of 5-7 cm in length was made along the hairline in the postauricular region. The occipital artery and the other superficial vessels were retracted to reach the calvarium. Subsequently, retrosigmoidal keyhole craniectomy was performed. The dura mater was opened to reach the pontocerebellar region. With the exception of one patient, the superior petrosal vein was sacrificed in all patients. Following visualisation of the trigeminal nerve, Teflon was implanted between it and the offending vessel, tissue glue was used, and the dura was sutured. Polymethyl methacrylate (PMMA), which was prepared and modelled manually as a bone graft, was a useful, cheap, natural, and easy technique to repair the calvarium following MVD surgery. We described the in situ cranioplasty technique in 2016 (5).

\section{RESULTS}

Of the 63 patients, 38 were female and 25 were male. The mean age was 48.6 years old (ranging from 23 to 81 ) in females and 50.8 years old in males (ranging from 26 to 85 ) (Table II). A total of 58 patients with sharp, stabbing, electrical pain were
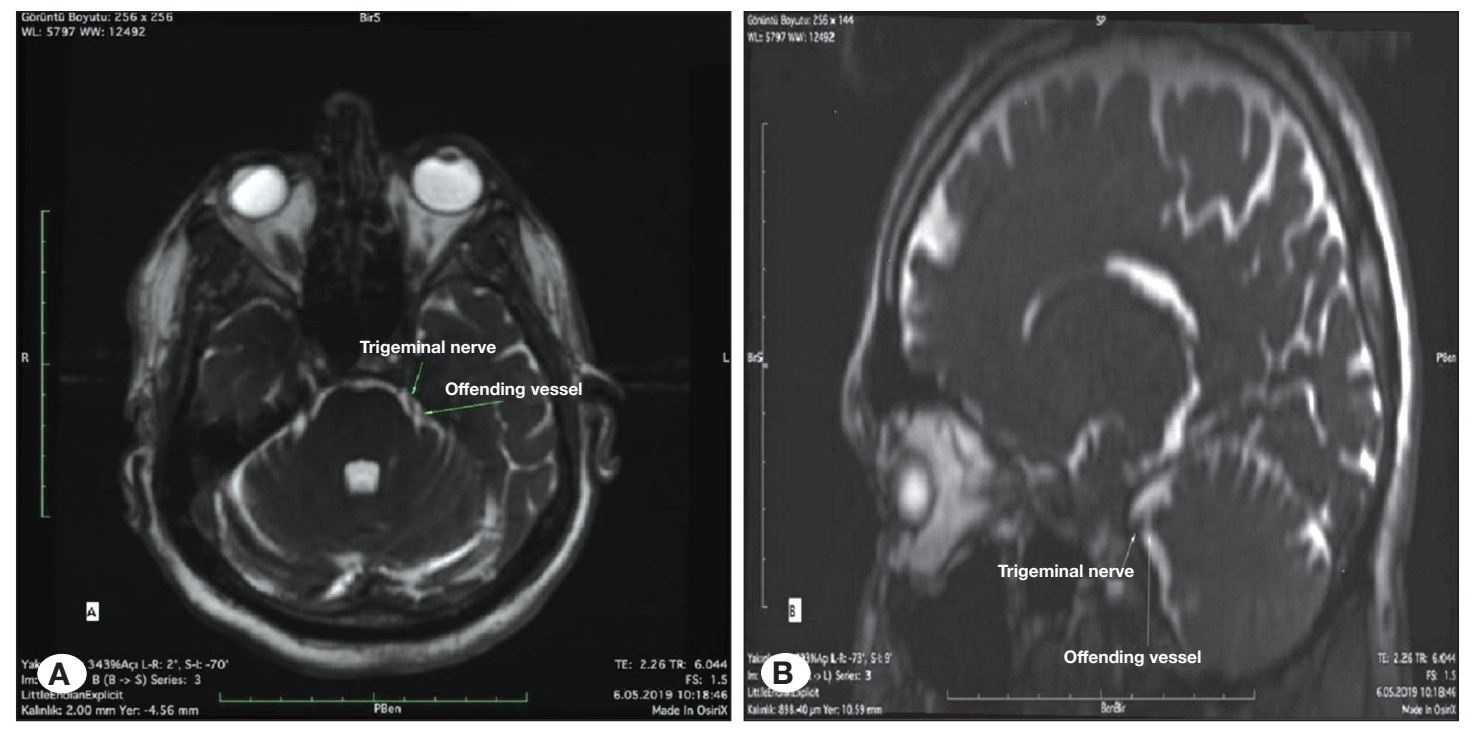

Figure 1: T2W MRI in axial (A) and sagittal (B) plane shows neurovascular conflict on left side (short arrow: trigeminal nerve, long arrow: offending vessel). 
considered as having typical trigeminal neuralgia (TN), while 5 patients with less intense, constant, dull or burning pain were considered as having atypical TN. Pain was felt on the right side in 36 patients $(57 \%)$ and on the left side in 27 patients (43\%). The patients' preoperative painful periods ranged from 1 to 30 years, with an average of 6 years. Pain was in V1 in

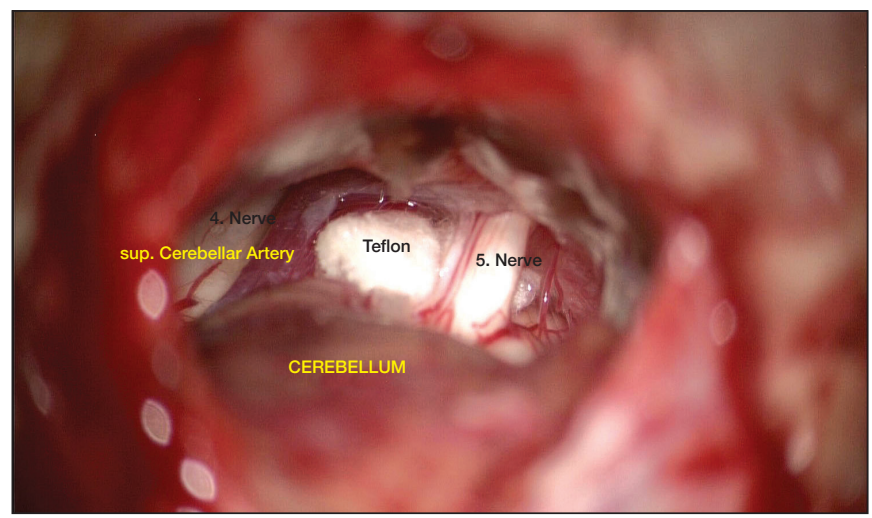

Figure 2: Intraoperative photograph shows trigeminal nerve $\left(5^{\text {th }}\right.$ nerve), superior cerebellar artery as the offending vessel and the teflon felt between them.

Table II: Demographic Characteristics of Patients

\begin{tabular}{lcc}
\hline Gender & Number of patients & Age (years) \\
\hline Female & 38 & 48.6 (range 23-81) \\
\hline Male & 25 & 50.8 (range 26-85) \\
\hline Total & 63 & 50.1 (range 23-85) \\
\hline
\end{tabular}

Table III: Pain Properties

\begin{tabular}{cc}
\hline Trigeminal neuralgia & Number of patients \\
\hline Typical & $58(92.1 \%)$ \\
\hline Atypical & $5(7.9 \%)$ \\
\hline Side of pain & Number of patients \\
\hline Right & $36(57 \%)$ \\
\hline Left & $27(43 \%)$ \\
\hline
\end{tabular}

Number of patients according to the affected branch of trigeminal nerve

\begin{tabular}{cccccc}
\hline V1 & V2 & V3 & V1-2 & V2-3 & V1-2-3 \\
\hline 1 & 1 & 5 & 1 & 50 & 5 \\
\hline
\end{tabular}

one patient, $\mathrm{V} 2$ in one patient, $\mathrm{V} 1-2$ in one patient, $\mathrm{V} 3$ in 5 patients, V2-3 in 50 patients, and in the V1-2-3 branches of the trigeminal nerve in 5 patients.

During surgery, neurovascular conflict was observed as arterial alone in 37 patients, venous structure alone in 14 patients, and both the artery and vein in 8 patients. In 4 cases, there was no neurovascular conflict. Vascular compression on the trigeminal nerve was: the anterior inferior cerebellar artery (AICA) in one patient, basilar artery (BA) in one patient, AICA and superior cerebellar artery (SCA) in two patients, SCA alone in 32 patients, vertebral artery (VA) in one patient, and a combination of SCA and venous structure in 7 cases (Table III). In one patient who had pain in all branches of the trigeminal nerve (V1-2-3), there was fusiform aneurysmatic dilatation, which causes compression on $7^{\text {th }}$ and $8^{\text {th }}$ cranial nerve and SCA compression on the trigeminal nerve. The most common cause of neurovascular conflict was the SCA, and the most common painful region was the V2-3 branches of the trigeminal nerve.

All patients suffered from severe pain despite medical treatment prior to surgery. Despite using at least two combined medications at a maximum dosage for an optimum period, almost all patients had ongoing pain. Surgical or ablation techniques had been previously applied in 18 (28.5\%) patients. One patient had multiple sclerosis and 19 (30.1\%) patients had hypertension.

Patients were questioned regarding the severity of their pain during the first month of the postoperative period, which is accepted as the early period, and after the first month, which is accepted as the long-term follow-up.

During the early postoperative period, the pain severity in 8 patients partially decreased but there were no changes in 2 patients. A total of 53 patients said their pain completely ended, but all of these patients were still receiving at least a single medication.

During the long-term follow-up, 9 patients who had no pain during the early postoperative period said that their pain started again but more mildly as compared with the status prior to surgery. In this group, one patient with atypical pain had been completely pain-free following the surgery, while the other patient with atypical pain did not benefit from the operation. The other three patients mentioned that they rarely felt short-term blunt pain only in the surgical area. During the long-term follow-up, the onset of pain ranged from 1 to 14 months. In one patient, the pain began 5 years after the operation.

Thus, $84 \%$ of patients were completely pain-free during the early postoperative period, while $16 \%$ still have ongoing pain

Number of patients according to the offending vessel

\begin{tabular}{cccccccc}
\hline SCA & AICA & BA & VA & AICA, SCA & VA, SCA, SPV & SCA, SPV & Venous structure alone \\
\hline 32 & 1 & 1 & 1 & 2 & 1 & 7 & 14 \\
\hline & Artery & & & Artery and Vein Combination \\
\hline
\end{tabular}


with reduced severity. In 2 patients (3.1\%), there was no change in the severity of pain. During the long-term follow-up, the pain-free status rate was $69.8 \%$ (Tables IV, V).

Three patients had cerebrospinal fluid (CSF) leakage. In two patients, surgery was not needed, while CSF fistula was repaired in one patient. One patient was re-operated on due to flap disposition. Wound infection, facial paralysis/ paraesthesia, hearing loss, seizure, delirium, transient ischaemic attack, epidural/subdural/intracranial haemorrhage, encephalomalacia, cortex damage, cardiac or respiratory problems, or death did not occur in any patient (Table VI).

Table IV: Post-Operative Pain Condition

\begin{tabular}{cccc}
\hline \multicolumn{2}{c}{ Early period } & \multicolumn{2}{c}{ Long term follow-up } \\
\hline Pain free & Not pain free & Pain free & Not pain free \\
\hline $53(84 \%)$ & $10(16 \%)$ & $44(69.8 \%)$ & $19(30.2 \%)$ \\
\hline
\end{tabular}

Table V: In Long Term Fallow-Up, Barrow Neurological Institute (BNI) Pain Intensity Scale Score

\begin{tabular}{cc}
\hline BNI Score & Number of patients \\
\hline I & $44(69.8 \%)$ \\
\hline II & $8(12.6 \%)$ \\
\hline III & $5(7.9 \%)$ \\
\hline IV & $4(6.3 \%)$ \\
\hline V & $2(3.1 \%)$ \\
\hline
\end{tabular}

Table VI: Surgical Complications

\begin{tabular}{ll}
\hline Complication & $\mathbf{n}(\%)$ \\
\hline BOS leakage & $3(4.7)$ \\
\hline Flap disposition & $1(1.6)$ \\
\hline Wound Infection & None \\
\hline Facial Paralysis/Paresthesia & None \\
\hline Hearing Loss & None \\
\hline Seizure, Delirium & None \\
\hline Transient ischemic attack /stroke & None \\
\hline Death & None
\end{tabular}

The 2 patients who did not benefit from the operation during the early postoperative period were both female. The patients' painful zone was the V2-3 branch of the trigeminal nerve and neither had any complications. One had right-sided typical pain and SCA compression on the trigeminal nerve near the pons, and the other had left-sided atypical pain and vein compression was present.

During the early postoperative period, 3 of the 8 patients who partially benefited from surgery without a completely pain-free condition were those whom had complications (37.5\%). One patient was re-operated on due to CSF leakage and the other due to flap disposition. In the third case of CSF leakage in the patient with multiple sclerosis, the leakage disappeared without surgery. Neurovascular conflict was seen in 7 of these: 3 had venous compression, the other 3 had SCA compression, and one had SCA and SPV compression on the trigeminal nerve.

In 9 patients who had been pain-free during the early postoperative period but had pain again during the longterm follow-up, there were no complications except for one $(11.1 \%)$ who had CSF leakage. Five of the 9 patients had SCA compression, one had venous compression, and two had SCA and venous compression on the trigeminal nerve. There was no compressive anatomical structure in the remaining patient but there was a history of surgery due to a parotid tumour. The average duration of illness was 7 years (ranging from 1 to 15 years), and the average age was 63.8 years old, which was above the overall average. Four patients had hypertension, one had atypical pain, and one had had a previous Gamma Knife procedure (Table VII).

\section{DISCUSSION}

TN is a chronic syndrome characterised by intense intermittent facial pain that ruins daily life. Washing the face, shaving, chewing, or any other tactile stimulation, even talking in some patients, can trigger pain attacks. Although the primary treatment for TN is medication therapy, most patients become refractory, at which point, MVD is the most effective treatment. Pain relief following surgery depends on many factors such as age, accompanying diseases, and neurovascular conflict, but mostly on surgical technique. Primarily, eligible patients should be chosen for MVD; those with neurovascular conflict and typical pain are more suitable for surgery. MRI is helpful for determining neurovascular conflict, with a positive predicting value of $95 \%$. In some cases, MRI shows bilateral conflict: on the symptomatic side $89 \%$ and on the asymptomatic side $78 \%$, but the most important is the painful side of the patient

Table VII: Comparison of the Patients with Pain Recurrence Who was Pain-Free in Early Postoperative Period (A) with the General Patient Group (G)

\begin{tabular}{cccccc}
\hline & $\begin{array}{c}\text { Mean age } \\
\text { (years) }\end{array}$ & $\begin{array}{c}\text { Duration of illness } \\
\text { (mean year) }\end{array}$ & $\begin{array}{c}\text { Hypertension } \\
(\%)\end{array}$ & $\begin{array}{c}\text { Early procedures } \\
\text { (\%) }\end{array}$ & $\begin{array}{c}\text { SCA compression } \\
\text { (\%) }\end{array}$ \\
\hline $\mathrm{A}$ & 63.8 & 7 & 44.4 & 11.1 & 55.5 \\
\hline $\mathrm{G}$ & 50.1 & 6 & 30.1 & 28.5 & 50.8 \\
\hline
\end{tabular}


$(8,12)$. In a large study reported by Tyler-Kabara et al., the rate of complete pain relief following MVD in typical TN was $84.1 \%$ and pain control after surgery was $98.2 \%$. These rates were recorded as $46.9 \%$ and $86.6 \%$, respectively, in atypical TN. Surgical outcomes suggest that patients with typical TN benefit from MVD surgery more than those with atypical TN $(10,15,16,19)$.

Besides MRI, exploration during surgery is valuable in determining the conflicting structure. While the common cause of neurovascular conflict is SCA, there are also accompanying venous structures in most cases. In an anatomical-surgical study by Dumot and Sindou in 326 patients who underwent MVD, 124 patients (38\%) had venous conflict; alone in 29 (8.9\%) and in conjunction with an artery in 95 (29.1\%) (3). Offending venous structures were divided into two groups: superficial and deep superior petrosal venous systems (sSPVS and dSPVS). It was reported that arterial compression is well established, but the role of the veins remains under discussion. Coagulation-division was performed on offending venous structures in $36.8 \%$, and cleavage was carried out in the remaining patients. Sacrification was only performed for one of the affluent SPVs, and the trunk was preserved. Overall in their series, one or more veins were sacrificed in 56 patients $(45.2 \%)$. Venous neurovascular conflict rates vary from $11.8 \%$ to $91.7 \%$ in the literature. Anatomical variation of veins is more common, such that it is easy to miss an offending vein and trigeminal root next to the porus if not examined carefully during surgery. Authors recommend avoiding sacrification of an affluent SPV, but it is complicated to identify the branches of SPVs during surgery in a limited time period. Moreover, not recognising a venous conflict ends with an inadequate decompression and recurrence, and once a conflicting venous structure is caught, a neurosurgeon should search the area carefully for more (3).

In some series, especially in the case of venous compression, the recurrence of pain seems to be more frequent due to disregard of the offending venous vessel, which ends up with inadequate decompression $(9,11,13)$. In our series, during the early period, 5 of 10 patients who did not fully recover from pain, and 3 of 9 patients whose pain recurred during the late period, had accompanying venous compression. However, it was reported in a series by Zhong et al., that if the cause of the compression is a large vein and it is the only vein that drains into the petrous sinus, it should be preserved, otherwise it can cause death (21). According to Dumot and Sindou, sSPVS should be preserved to avoid cerebellar swelling and infarction, with exploration of the trigeminal root being recommended by passing through the SPV, limiting sacrification to one affluent SPV (3).

When patients were questioned regarding their recurrent pain type, they described a neuralgiform pain like the TN pain they used to have, not a new kind such as benign intracranial hypertension or other pain types.

In addition to vascular compression, in diseases such as multiple sclerosis (MS), demyelination of the trigeminal nerve can result in TN. In patients with MS, the TN incidence rate is $2-4 \%$ and the disease can be partially refractory to all procedures (18). We also describe a case of MS with partially decreased postoperative pain that increased again during the late period. In a review by Ariai et al., it was reported that success of MVD surgery in TN patients with MS is lower than that in the idiopathic group. Patients who recovered from pain during the early period following surgery developed pain again during the late period (1). Although the surgical success rates vary according to the center, Nunta-Aree et al., reported that during the early postoperative period, the painfree rate was $67.3 \%$, while during the following two years, it became $61.5 \%$. According to our results, the pain-free rate was $84 \%$ during the early period and $69.8 \%$ during the late period. It has been noted that pain in atypical TN cases is partly more robust. Complications of MVD surgery are hearing loss, facial hypoesthesia, facial paralysis, temporary ataxia, central nervous system infections, wound infections, cerebral haemorrhage, CSF leakage, or death. The most common complications are temporary facial hypoesthesia, CSF leakage, and wound infections $(14,20)$. The complication rate in our series was $6.3 \%$, and the most common complication was CSF leakage, which recovered without surgery in 2 of 3 cases. The success rate that we achieved correlates with other reports in the literature, and no serious complications or death were seen.

In our series, with the exception of one case, the SPV was sacrificed and no complications were seen as a result. With a limited time period during surgery, it is important to achieve a full decompression. Venous structures mostly go unnoticed, since the SPV trigeminal region cannot be scanned well; thus, we generally sacrifice SPV in all patients as our special technique. However, more experience is needed for better results.

\section{CONCLUSION}

MVD is the most effective method in appropriate cases for the treatment of TN. Besides careful preoperative evaluation, the most important issue that decides surgical achievement is the surgical technique and the experience of the neurosurgical team. In the present review, we claim that SPV sacrification can be performed in most cases and is not related to gross complications; therefore, venous sacrification is not associated with pain recurrence. Further studies are needed to better assess surgical approaches in TN.

\section{- REFERENCES}

1. Ariai MS, Mallory GW, Pollock BE: Outcomes after microvascular decompression for patients with trigeminal neuralgia and suspected multiple sclerosis. World Neurosurgery 81(34): 599-603, 2014

2. Barker FG, Jannetta PJ, Bissonette DJ, Larkins MV, Jho HD: The long-term outcome of microvascular decompression for trigeminal neuralgia. The New England Journal of Medicine 334:1077-1083, 1996

3. Dumot C, Sindou M: Trigeminal neuralgia due to neurovascular conflicts from venous origin: An anatomical-surgical study (consecutive series of 124 operated cases). Acta Neurochirurgica (Wien) 157(3):445-466, 2015 
4. Elias WJ, Burchiel KJ: Microvascular decompression. The Clinical Journal of Pain 18:35-41, 2002

5. Erbas YC, Pusat S, Atac GK, Erdogan E: In-situ cranioplasty after microvascular decompression: A technical note. Turk Neurosurgery 27(3):479-481, 2017

6. Erdogan E, Civelek E, Onal MB, Solmaz I, Kural C, Yakupoglu $\mathrm{H}$ : A new method of patient's head positioning in suboccipital retrosigmoid approach. Neurol India 57(6):777-779, 2009

7. Frederickson AM, Gold MS, Sekula RF Jr: Pathogenesis of trigeminal neuralgia. In: Li ST, Zhong J, Sekula RF Jr (eds), Microvascular Decompression Surgery. Springer, 2006:59-66

8. Hitchon PW, Bathla G, Moritani T, Holland MT, Noeller J, Nourski KV: Predictability of vascular conflict by MRI in trigeminal neuralgia. Clinical Neurology Neurosurgery 182:171-176, 2019

9. Hong W, Zheng X, Wu Z, Li X, Wang X, Li Y, Zhang W, Zhong $J$, Hua $X$, Li S: Clinical features and surgical treatment of trigeminal neuralgia caused solely by venous compression. Acta Neurochirurgica (Wien) 153:1037-1042, 2011

10. Kabatas S, Karasu A, Civelek E, Sabanci AP, Hepgul KT, Teng YD: Microvascular decompression as a surgical management for trigeminal neuralgia: Long-term follow-up and review of the literature. Neurosurgery Review 32:87-93, 2009

11. Kimura T, Sako K, Tohyama Y, Yonemasu Y: Trigeminal neuralgia caused by compression from petrosal vein transfixing the nerve. Acta Neurochirurgica (Wien) 141:437,438, 1999

12. Maarbjerg S, Wolfram F, Gozalov A, Olesen J, Bendtsen L: Significance of neurovascular contact in classical trigeminal neuralgia. Brain 138:311-319, 2015

13. Matsushima T, Huynh-Le P, Miyazono M: Trigeminal neuralgia caused by venous compression. Neurosurgery 55:334-337, 2004

14. Nunta-Aree S, Patiwech K, Sitthinamsuwan B: Microvascular decompression for treatment of trigeminal neuralgia: Factors that predict complete pain relief and study of efficacy and safety in older patients. World Neurosurgery 110:979-988, 2018
15. Osburn LL, Moller AR, Bhatt JR, Cohen-Gadol AA: Hemilingual spasm: Defining a new entity, its electrophysiological correlates and surgical treatment through microvascular decompression. Neurosurgery 67:192-195, 2010

16. Ray DK, Bahgat D, McCartney S, Burchiel KJ: Surgical outcome and improvement in quality of life after microvascular decompression for hemifacial spasms: A case series assessment using a validated disease-specific scale. Stereotactic Functional Neurosurgery 88:383-389, 2010

17. Rogers CL, Shetter AG, Fiedler JA, Smith KA, Han PP, Speiser $\mathrm{BL}$ : Gamma knife radiosurgery for trigeminal neuralgia: The initial experience of The Barrow Neurological Institute. Int $\mathrm{J}$ Radiat Oncol Biol Phys 47:1013-1019, 2000

18. Sarlani E, Grace EG, Balciunas BA, Schwartz AH: Trigeminal neuralgia in a patient with multiple sclerosis and chronic inflammatory demyelinating polyneuropathy. Journal of the American Dental Association 136 (4):469-476, 2005

19. Tyler-Kabara EC, Kassam AB, Horowitz MH, Urgo L, Hadjipanayis C, Levy El, Chang YF: Predictors of outcome in surgically managed patients with typical and atypical trigeminal neuralgia: comparison of results following microvascular decompression. Journal of Neurosurgery 96:527-531, 2002

20. Xiang H, Wu G, Ouyang J, Liu R: Prospective study of neuroendoscopy versus microscopy: 213 cases of microvascular decompression for trigeminal neuralgia performed by one neurosurgeon. World Neurosurgery 111:335-339, 2018

21. Zhong J, Li ST, Zhu J, Guan HX, Zhou QM, Jiao W, Ying TT, Yang XS, Zhan WC, Hua XM: A clinical analysis on microvascular decompression surgery in a series of 3000 cases. Clin Neurol Neurosurg 114: 846-851, 2012 